\title{
Research in progress-electronic patient records: a new era
}

\author{
D Peckham, ${ }^{1}$ P Whitaker, ${ }^{1} \mathrm{H}$ White ${ }^{2}$
}

${ }^{1}$ Leeds Centre for Cystic

Fibrosis, St James's University Hospital, Leeds, UK

${ }^{2}$ Faculty of Health and Social Science, Leeds Beckett University, Leeds, UK

\section{Correspondence to} Dr Daniel Peckham, Leeds Centre for Cystic Fibrosis, St James's University Hospital, Beckett Street, Leeds LS9 7TF, UK; daniel.peckham@nhs.net

Received 18 November 2014 Accepted 19 November 2014 Published Online First 10 December 2014

\begin{abstract}
Clinical information systems and electronic records are starting to appear in secondary care and herald new potentials for improving health provision and capturing high quality data. In 2006, we set up a program to develop electronic patient records (EPR) for chronic disease using Cystic Fibrosis (CF) as our initial model. Seven years on we are now exploring the real time clinical data to identify risks, trends and outcomes in chronic disease management. We are also working to establish new models of integration and to connect information between the client and all areas of health care.
\end{abstract}

Handwritten and typed paper records of patients under the care of the cystic fibrosis (CF) unit in Leeds have been replaced by electronic patient records (EPRs). This report describes the steps required to design and implement an EPR system, and outlines the rationale and potential benefits of such a transition. Although introduced for use in CF, we have modified and deployed a system that we believe could have wider use in medicine.

To work effectively, the shift from paper to EPR needed to be clinically driven and involve all members of the clinical team. We wanted to avoid an unrealistic quest for total perfection, to ensure that the aims were achievable. Constant development and upgrading remains essential and the system needs to be generic and capable of use in other settings.

Standardised information generated in real time through EPR is proving to be an invaluable source of longitudinal data with the potential to shed new light on natural history in different subsets of patients and to allow treatment to be assessed in relation to specific clinical and biological factors. In addition to the clinical and research advantages, EPR as presented here is a powerful valuable tool for audit and for economic and other administrative purposes.

\section{THE OLD WAY AND 'ONE CLICK OF THE MOUSE'}

Prior to the shift to EPR, patients attended for full-day assessment in an annual review. The results were handwritten, a summary dictated by the consultant and typed by medical secretaries. Clinic letters and discharge summaries were dictated and typed. The paper records arising from these various aspects of care were held in a boxed filing system. Unlike the old way, there is now a continuous record of each patient's information which is easily accessed by 'one click of the mouse'.

The impact of the EPR system on clinical care has been dramatic. ${ }^{1}$ In a blind survey, staff strongly approved EPR and had no wish to return to paper records. ${ }^{1}$ Multidisciplinary communication was improved and there was general agreement that EPR had resulted in the earlier detection of clinical deterioration. Of particular value have been the alerts built into the system relating to treatment, investigations, attendance, drug allergies and drug interactions.

\section{CAPTURING THE COMPLEXITY OF DISEASE}

An understanding of the intricate nature of $\mathrm{CF}$ as a chronic disease and the limitation of diseasespecific data systems in managing multiple co-morbidities is essential to the design and practical use of EPR. A key tenet was the realisation that any electronic record should be generic and configurable to other diseases. Our system, for example, has encompassed the management of non-CF complications such as myocardial and cerebral infarcts, subarachnoid haemorrhage, malignancies and epilepsy.

Information captured at this level of detail needs to be in a format that is standardised, relevant and capable of replication by other units. This required the writing of codes, each of which encompassed specific clinical, investigational and biological facets of CF including gene mutations and microbiology recording of each cultured isolate. To address this need, more than 500 new codes were developed by one of us (DP). Importantly, these local codes have been migrated and mapped to the international system Snomed-CT (sysematised nomenclature of medicine-clinical terms) (http://www.ihtsdo.org/ snomed-ct).

\section{CHALLENGES AND PITFALLS}

Developing EPR in 2007 proved difficult due to the absence of appropriate hardware and hospital infrastructure. We even managed to crash the link between Seacroft and St James's Hospitals during initial software download! As a result, the CF units had to fund the installation of ethernet cabling as well as of all the computers and printers. There was also an absence of hospital IT support at the start of the project, which significantly increased our workload and could have resulted in project failure. Because the project was outside the scope of the overall Trust strategy, support was absent even though the project was cost-effective, innovative and offered the prospect of delivering an improved clinical service supported by all staff. Things have changed, and as a result of the success of the project, support is now forthcoming.

The Achilles heel of a stand-alone system is the absence of true hospital integration. As a result, Trust IT departments often adopt a one-solutionfits-all strategy, which may hinder progress in the 
long run. It is our view that healthcare professionals should be able to adopt the best type of solution that can address specific healthcare needs. Integration between systems is crucial for long-term success, and while many solutions are available, new ones can be developed. Work is ongoing to link our EPR to generic hospital portals and patient administration systems.

\section{THE SYSTEM IN OPERATION}

Since 2007, we have stopped using paper records. Live data (continuously backed up) are displayed on computer screens and can be accessed by each member of the clinical team using secure passwords. Staff in seminar room clinical meetings, multidisciplinary team meetings and on ward rounds access EPR as a basis for decision making. The system is schematised in figure 1.

Seven years on, the system has evolved and migrated to a new more powerful platform. We now have a configurable, intelligent graphical interface that tailors the way information is presented to both clinicians and patients (figure 2). Surveillance, epidemiological and continuous phenotypical data have allowed us to see patterns and trends in longitudinal data. For example, the H1N1 influenza pandemic in 2009 highlighted the importance of global health surveillance and triggered an unparalleled response with the widespread uptake of flu vaccination and anti-viral medication. Prior to this, we had instituted routine testing for respiratory viruses in acute pulmonary exacerbations with each isolate documented in the EPR. ${ }^{2} 3$ The surveillance data available through EPR indicated in 2009 that the vast majority of patients presenting to hospital with acute viral infection presumed to be $\mathrm{H} 1 \mathrm{~N} 1$, in fact, had rhinovirus infection. It was only in the following year that the H1N1 pandemic took hold and resulted in greater morbidity.

One aspect of our research has been directed at understanding the multitude of factors that influence disease progression and to explore real-time clinical data to identify risks, trends and outcome in chronic disease management. The display through EPR of data in graphical form can help cognitively by making patterns of disease more obvious.

Using EPR, a number of associations with disease progression have begun to emerge. For example, it was observed that the fluctuation of random blood glucose measurements appeared to differ between patients, even in those with normal glucose tolerance. While such values had been recorded for many years prior to EPR, no pattern had been identified. However, our data suggested that there was a significant difference in the distribution of blood glucose levels and median HbA1c values in the 3 years preceding a diagnosis of cystic fibrosis-related diabetes (CFRD), compared with patients who do not develop CFRD (unpublished).

As part of a recent adherence study, we investigated predictive markers of adherence. From clinical experience and exposure to graphical representations of data, we hypothesised that there was a difference in the fluctuation of both $\mathrm{FEV}_{1}$ and $\mathrm{C}$ reactive protein (CRP) in patients who were less adherent to treatment

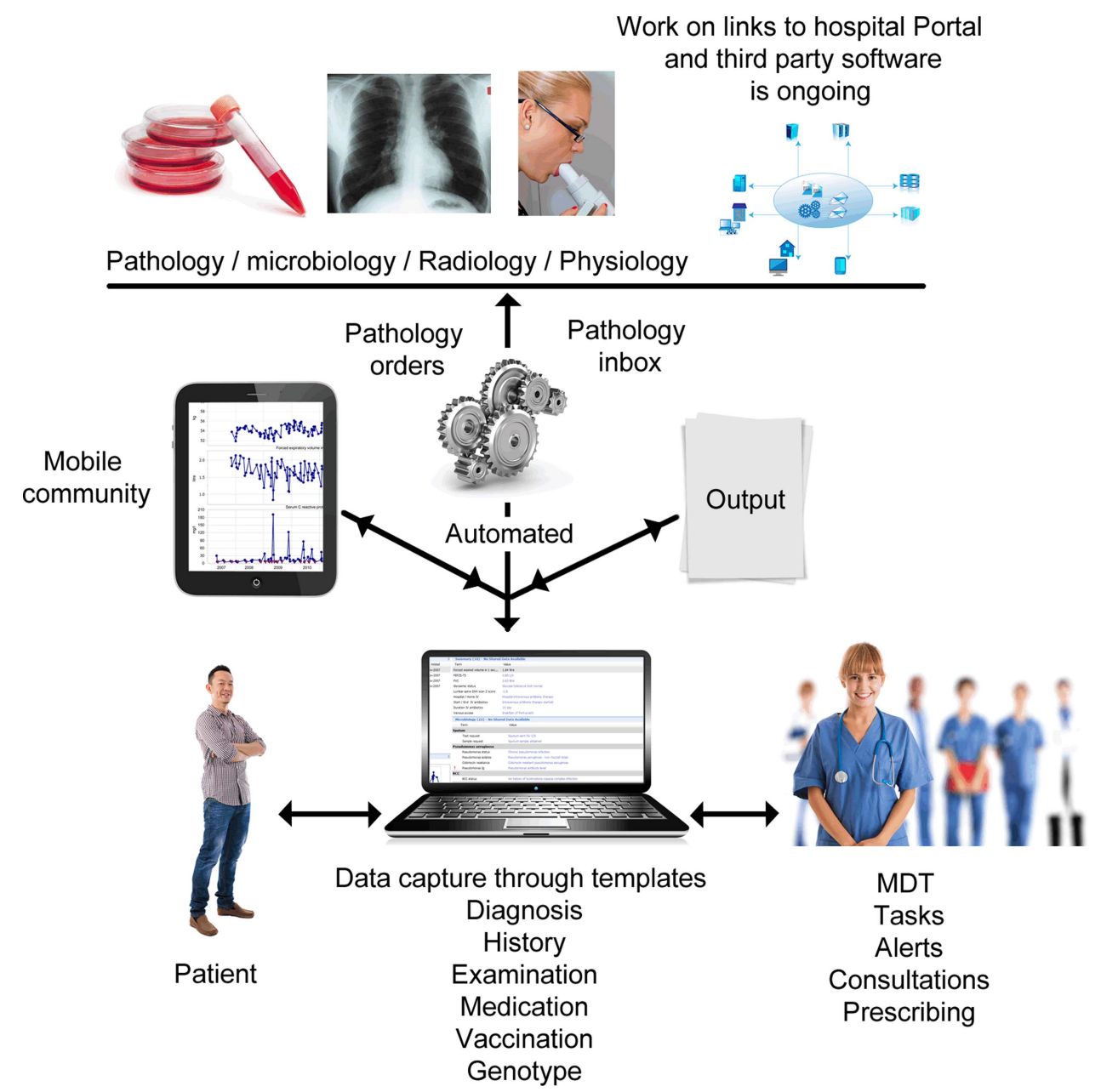

Figure 1 Schema of EPR in cystic fibrosis. EPR, electronic patient record; MDT, multidisciplinary teams. 


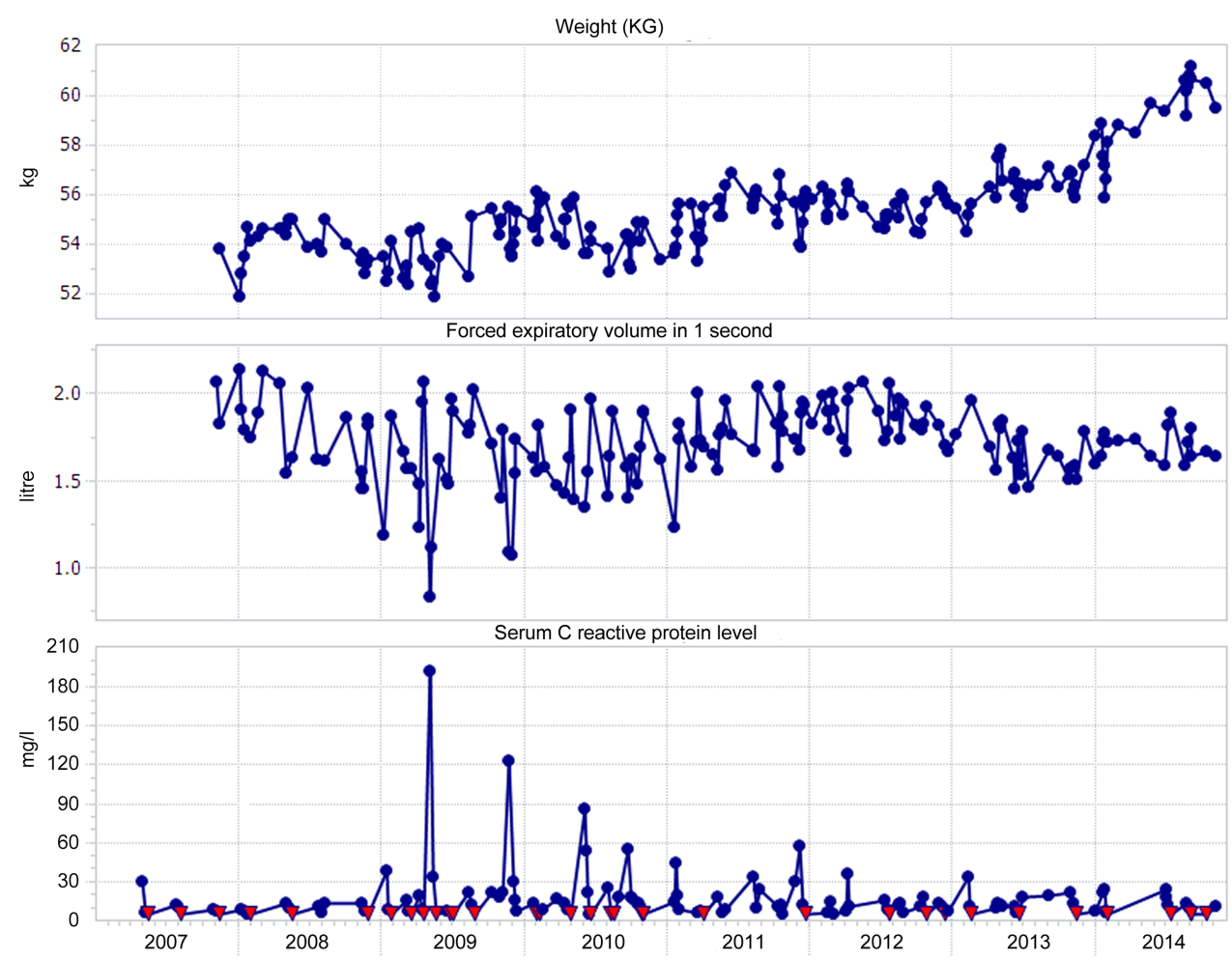

Figure 2 Example screen shot of 'one click of the mouse' summary page generated during a consultation. Significant fluctuations in lung function, weight and $\mathrm{C}$ reactive protein in a patient with non-adherence to physiotherapy and medication. Dramatic improvement in weight and reduction in variability of each parameter following the start of a long-term relationship.

(figure 2). Electronic clinical records were examined to extract all longitudinal measures of $\mathrm{FEV}_{1}$, weight and CRP recorded at each clinical contact in the previous year. Coefficients of variation $(\mathrm{CoV})$ were calculated to assess the degree of variation in each measure over that time period. Preliminary results showed that self-reported adherence was inversely related to $\mathrm{CoV} \mathrm{FEV}_{1}$ and that there was a weak relationship between self-reported adherence and CoV CRP. ${ }^{4}$

\section{WAY FORWARD}

What has been set in place is primarily a clinical management system, although the availability of longitudinal data can answer some research questions. The next phase of the project will extend the use of EPR as a research as well as a clinical tool. Plans are in hand to link with CF units in different geographical locations within a collaborative network in order to enable analyses of data on larger numbers of patients with standardised records and to recruit patients with well-defined attributes into clinical trials. The accrual of larger numbers of patients will also allow the analysis of smaller subsets within the CF population-an undertaking which is unlikely to be possible at the level of individual units. Genotypical information has been included in EPR from the outset and we are exploring methods for data linkage with genomics.

Ongoing projects include automating the flow of information between primary, community and secondary care and the transfer of the experience gained with EPR in CF to other diseases.
In addition, we are exploring the provision of patient access to their electronic health records.

These projects are challenging and ambitious, but their successful realisation will provide a model for the wider use of electronic data capture and demonstrate how it might be integrated within healthcare at all levels for the benefit of patients, professionals and wider society, a panacea which we all long for.

Contributors DP conceived the project, developed the codes, modified, deployed and manages the electronic patient record. HW is the PI on the adherence study and collaborated closely with DP. PW participated in clinical development and is leading the TB EPR.

Competing interests DP has undertaken development work for Egton Medical Information Systems.

Provenance and peer review Not commissioned; internally peer reviewed.

\section{REFERENCES}

1 Peckham D, Etherington C, White $\mathrm{H}$, et al. The development and deployment of integrated electronic care records in a regional adult and paediatric cystic fibrosis unit. J Cyst Fibros 2014;13:681-6.

2 Etherington C, Naseer R, Conway SP, et al. The role of respiratory viruses in adult patients with cystic fibrosis receiving intravenous antibiotics for a pulmonary exacerbation. J Cyst Fibros 2014;13:49-55.

3 Whitaker $\mathrm{P}$, Etherington $\mathrm{C}$, Denton $\mathrm{M}$, et al. A/H1N1 flu pandemic. A/H1N1 and other viruses affecting cystic fibrosis. BMJ 2009;339:b3958.

4 Denman S, White H, Shaw N, et al. Objective predictors of self-report of adherence in adults with cystic fibrosis. J Cyst Fibros 2014;13:S22. 